\title{
Levulinic acid: a novel sustainable solvent for lignin dissolution
}

2 Elodie Melro ${ }^{a},{ }^{*}$, Alexandra Filipe ${ }^{b}$, Artur J. M. Valente ${ }^{a}$, Filipe E. Antunes $^{\mathrm{a}}$, Anabela Romanoc,

3 Magnus Norgren ${ }^{d}$ and Bruno Medronho ${ }^{c, d}$

4

5 a CQC, Department of Chemistry, University of Coimbra, Rua Larga, 3004-535 Coimbra, Portugal

$6{ }^{b}$ CIEPQPF, Department of Chemical Engineering, University of Coimbra, Pólo II - R. Silvio Lima, 3030-790

7 Coimbra, Portugal

8 'MED - Mediterranean Institute for Agriculture, Environment and Development, University of Algarve, Faculty

9 of Sciences and Technology, Campus de Gambelas, Ed. 8, 8005-139 Faro, Portugal

$10 \quad{ }^{d} F S C N$, Surface and Colloid Engineering, Mid Sweden University, SE-851 70 Sundsvall, Sweden

$12 *$ Corresponding author

13 E-mail addresses: elodie.melro@uc.pt (Elodie Melro)

\section{Abstract}

16 Lignin is a natural macromolecule with potential to be used as a fossil resource to produce

17 fuels, value-added chemicals, and novel biomaterials and composites. Due to its complex 18 structure, its efficient dissolution is still challenging, which hinders the applicability of this 19 natural resource at larger scale. The challenge becomes harder considering the current need 20 of sustainable and environmentally friendly solvents. This work reports, for the first time, the

21 dissolution of lignin in levulinic acid, a solvent that can be produced from renewable sources,

22 and compares its efficiency with common carboxylic acids and sulfuric acid. Levulinic acid 23 was found to dissolve 40 wt. \% kraft lignin; remarkably, the dissolution efficiency was 
24 maintained when adding up to $40 \mathrm{wt} \%$ of water. The addition of water decreases the

25 concentration of levulinic acid and the viscosity of the solutions formed which is benefic for

26 large-scale processing. The Kamlet-Taft $\pi^{*}$ parameter of the different acidic solvents was

27 estimated and found to correlate well with their solubility performance. Lignins previously

28 dissolved in levulinic and formic acids were selected to be regenerated and minor differences

29 were found in the content of functional groups, thermal stability, and their morphological

30 structure, when compared to native kraft lignin. The high dissolution performance and

31 favorable sustainable features makes this solvent highly appealing for future large-scale

32 dissolution and manipulation of lignin into novel biomaterials.

\section{Introduction}

35 The concern to reduce the consumption and dependence of fossil fuels has led to an increase

36 in the demand for products manufactured from renewable and sustainable sources, such as

37 lignin. Lignin is an amorphous, polyphenolic polymer, being the second most abundant 38 renewable biomaterial on Earth [1]. Today, the main source of lignin available is isolated from

39 spent pulping liquors produced after chemical pulping of wood. By far, the most important

40 pulping methods are kraft and sulfite cooking. The content of the most important functional

41 groups presents in the lignin materials, such as the phenolic hydroxyl, carboxyl, and sulfonate

42 groups, varies with the pulping process applied. Due to its high carbon content, high thermal

43 stability, biodegradability, antioxidant activity and favorable stiffness, there is a great interest

44 of using lignin to produce value-added products for various applications, such as

45 reinforcement fillers, antioxidants, UV adsorbents, antimicrobial agents, carbon fibre precursors, 
46 and biomaterials for tissue engineering and gene therapy [2,3]. The solubility in the water-based

47 cooking liquors is governed by the number of dissociating groups available in the lignin and

$48 \mathrm{pH}$ values approaching and exceeding the pKa value of the dissociating functional group [4-

49 6]. For example, in kraft lignins the pKa value for the main dissociating phenolic moieties is

50 close to 10, while in sulfite lignins the sulfonate groups have pKa's around 1-2. Like for other

51 polymers, the polydispersity introduces differences in the solubility characteristics within the

52 size distribution, which make low molecular fragments much easier to dissolve than larger

53 molecules. The reason is that the lower the molecular weight, the stringer is the entropic

54 driving force contribution for dissolution. These considerations refer to the translational

55 entropy of mixing. Moreover, due to the most frequent depolymerization reaction in kraft

56 cooking, the number of free phenolic groups increases when the molecular mass decreases

57 [7]. It has been found that it is possible to re-dissolve aggregated lignin if certain surface

58 active additives are introduced to a lignin dispersion; small additions of bile acid salts have

59 been shown especially efficient to re-dissolve kraft lignin in aqueous solutions[8]. The effect

60 of bile salts can, most likely, be assigned to an association at hydrophobic sites in the lignin

61 structure, which then increases the hydrophilicity of the lignin. Moreover, in water the

62 dissolution and colloidal stability of lignin can be increased by additions of certain

63 electrolytes (so called "salting in" effect), which shows that ion specific effects are also

64 important for solvency [9].

65 The ideal scenario would be the development of a solvent that, besides being highly efficient

66 and easily tuned to fit different types of lignin, should be also inexpensive, nontoxic, low

67 volatility, and viscosity [10]. In this respect, the use of sustainable solvents, such as, $\mathbf{Y}^{-}$ 
68 valerolactone and levulinic acid, can represent an interesting approach for lignin dissolution

69 [11]. $\mathrm{y}$-valerolactone has already been successfully included in binary solvent mixtures with

70 water, dimethyl sulfoxide, and dimethylformamide for lignin dissolution [10]. The amount of

71 lignin that can be dissolved in these $\gamma$-valerolactone-based mixtures was greater than most

72 common organic solvents [13]. However, at the best of our knowledge, the use of levulinic

73 acid as a direct solvent for lignin dissolution has never been described. This green organic

74 compound, has ketone and carboxylic groups which provides a wide range of functionality

75 and reactivity [16]. Levulinic acid has been used in many applications, such as textile dying,

76 pharmaceutical products, antifreezing agent, resin, food flavoring additive, coating material,

77 and animal feed [17]. Despite nowadays the use of renewable materials still carry reasonable

78 expensive precursors, low yields, and lack of efficient separation procedures [18], there is a

79 growing interest on this "green" acid. Levulinic acid has a large potential to be obtained from

80 different renewable sources, such as sugars (e.g., glucose, sucrose and fructose) and biomass

81 (e.g., wood, starch and agricultural wastes), through different dehydrating acid treatment 82 [19].

83 In this work, we intend to evaluate the performance of levulinic acid as a direct dissolution

84 system for lignin and shed light on the mechanisms involved in lignin dissolution. Therefore

85 Kraft lignin was dissolved with levulinic acid, and a set different of carboxylic acids (e.g. 2-

86 methylbutyric, 2-methylvaleric acid, glacial acetic acid and propionic acid) and a strong

87 inorganic acid (i.e. sulfuric acid), and their dissolution efficiency was compared. The effect of

88 diluting levulinic acid with water is also evaluated in regard of its dissolution performance.

89 The Kamlet-Taft $\pi^{*}$ parameter was estimated for the dissolution systems and correlated with 
90 lignin solubility. Additionally, lignins dissolved in levulinic and formic acids were regenerated

91 and analyzed by infrared spectroscopy, thermogravimetry and scanning electron

92 microscopy.

93

94 2. Materials and methods

95

96

2.1. Materials

97 Kraft lignin (selected as the model lignin) and 2-methylbutyric acid were purchased from

98 Sigma-Aldrich. Levulinic and formic acids were obtained from Merck and 2-methylvaleric acid

99 from Lancaster Synthesis. Acetic acid glacial and sulfuric acid were acquired from Panreac

100 while the propionic acid was obtained from Honeywell - Fluka. Sodium hydroxide ( $\mathrm{NaOH})$ was

101 purchased from José Manuel Gomes dos Santos, Lda., (Porto, Portugal). The 4-nitroanisol

102 (4NA) was acquired from Dagma. All the chemicals were used as received. The chemical

103 structures of different acids are depicted in the Figure 1. 
<smiles>O=CO</smiles>

formic acid

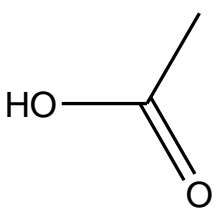

acetic acid

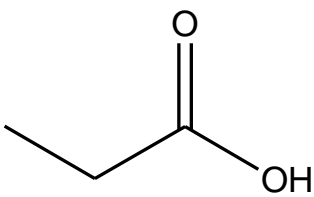

propionic acid

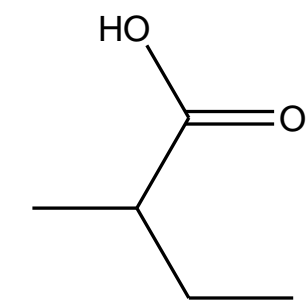

2-methylbutyric acid

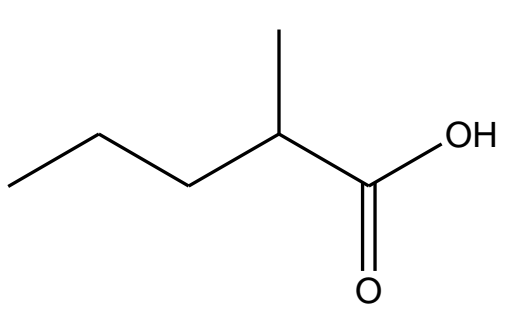

2-methylvaleric acid

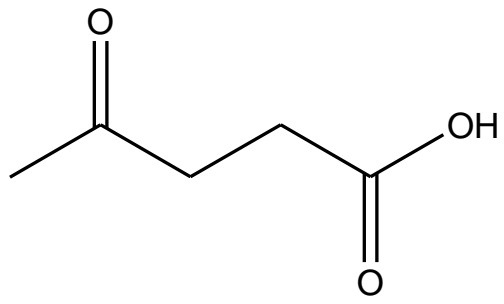

levulinic acid<smiles>O=S(=O)(O)O</smiles>

sulfuric acid

105 Figure 1. Molecular structures of the acids used.

106

\subsection{Dissolution efficiency of lignin}

Dispersions of kraft lignin were stirred with a magnetic stirrer for $24 \mathrm{~h}$ at 25,40 and $60^{\circ} \mathrm{C}$.

After centrifugation for $1 \mathrm{~h}$ at $14000 \mathrm{rpm}$ (17968 g), the supernatant was removed and

111 amount of lignin was estimated by measuring the absorbance at $288 \mathrm{~nm}$ using a UV-Vis

112 spectrophotometer (Shimadzu UV-2450). Calibration curves (i.e. nine solutions with

113 concentrations from 0.0001 to 0.004 wt. \% kraft lignin in 1 wt. \% NaOH(aq.) solvent) were

114 previously prepared. Samples were left to equilibrate and inspected over time by naked eye 115 and under optical microscopy (Linkam LTS 120 microscope equipped with a Q imaging station 116 (Qicam) Fast 1394 camera). 


\subsection{Solvatochromic Kamlet-Taft measurements}

119 The solvatochromic probe 4-nitroanisol (4NA) was used to estimate the Kamlet-Taft 120 parameter $\pi^{*}$ (polarizability index). A stock solution of the dye in DCM was prepared to a

121 concentration of $4 \mathrm{mM}$. The dye stock solution was added to the solvents under study to a 122 final concentration of $0.1 \mathrm{mM}$, that enables to obtain the absorbance values within the 123 required measurable range, and DCM was removed by evaporation at room temperature.

124 The absorption spectra of the solvatochromic probe was recorded from $250 \mathrm{~nm}$ to $500 \mathrm{~nm}$ 125 in a quartz cell with a $10 \mathrm{~mm}$ path length, using a Shimadzu UV/Vis spectrometer UV-1700 126 at $1 \mathrm{~nm}$ stepwise. The wavelength at maximum absorption $v_{\max }$ was determined for the 127 probe in each solvent. The $\pi *$ parameter was determined according to Kamlet and Taft [20], 128 using the equation:

$$
\pi^{*}=\frac{\left(34.12-v_{\max }\right)}{2.343}
$$

130 The constant values were obtained from multiple correlation equations that set $\pi^{*}$ at zero

131 for cyclohexane and unity for dimethyl sulfoxide [21].

\subsection{Rheometry}

134 The rheological measurements were carried out on a Haake Mars III (Thermo Fisher 135 Scientific) set with the cone-plate geometry $\left(1^{\circ}, 35 \mathrm{~mm}, 0.052 \mathrm{~mm}\right.$ gap). A Peltier unit was 136 used to ensure strict temperature control, which was set to $20.0 \pm 0.1 \stackrel{\circ}{ } \mathrm{C}$. The Newtonian 137 viscosity was accessed by nonlinear rotational tests from 0.1 to $50 \mathrm{~Pa}$.

\subsection{Fourier transform infrared spectroscopy (FTIR)}


140 Infrared spectra were recorded with a Thermo Nicolet 380 FT-IR apparatus (Thermo

141 Scientific) equipped with Smart Orbit Diamond ATR system. The FTIR analysis was performed

142 within the wave number range of $4000-400 \mathrm{~cm}^{-1}$. A total of 68 scans were used for each

143 spectrum at a resolution of $8 \mathrm{~cm}^{-1}$ in the absorbance mode. The background spectrum was

144 subtracted before each analysis. All the samples were freeze-dried before FTIR assays.

145

146

\subsection{Thermogravimetric analysis (TGA)}

147 Thermograms were measured using a thermogravimetric analyzer, TG 209 F Tarsus (Netzsch 148 Instruments). Previously lyophilized samples were weighed in aluminum pans (ca. 3 mg) and 149 subsequently heated from 25 to $1000 \stackrel{\circ}{\mathrm{C}}$ at a heating rate of $10 \stackrel{\circ}{ } \mathrm{C} \cdot \mathrm{min}^{-1}$ under $\mathrm{N}_{2}$ 150 atmosphere (flow rate of $50 \mathrm{~mL} \cdot \mathrm{min}^{-1}$ ).

151

\subsection{Scanning electron microscopy (SEM)}

153 A VEGA3 SBH from TESCAN scanning electron microscope equipped with a selected energy 154 dispersive X-ray microanalyser (EDX) was used to access the microstructural features of the 155 regenerated lignins. Before the SEM analysis, the samples were freeze dried, deposited 156 directly over the carbon tape on the support and sputtered with an approximately $6 \mathrm{~nm}$ thin $157 \mathrm{Au} / \mathrm{Pd}$ film, by cathodic pulverization using a SPI Module Sputter Coater, during $90 \mathrm{~s}$ at a 158 current of $15 \mathrm{~mA}$. The accelerating voltage used ranged from 5 to $15 \mathrm{kV}$, and the work 159 distance (WD) was set to $10 \mathrm{~mm}$. 


\subsection{Dissolution efficiency and Kamlet-Taft parameters}

164 The efficiency of levulinic acid to dissolve $10 \mathrm{wt}$. \% kraft lignin was evaluated and compared

165 with five carboxylic acids and sulfuric acid. For all carboxylic acids, an increase of dissolution

166 efficiency of lignin is verified with the reduction of the carbon chain length (Figure 2). A

167 similar trend was previously found for alcohols [22]. Surprisingly, levulinic acid does not

168 follow this trend since, despite having a long carbon chain, it can dissolve up to $40 \mathrm{wt}$. \% kraft

169 lignin. This sustainable solvent shows a similar dissolution performance to formic acid, but

170 much higher than the carboxylic acids of similar chain length (e.g., 2-methylbutyric acid). This

171 higher solubility efficiency might be due to the presence of an additional ketone group in the

172 levulinic acid structure. The sulfuric acid was able to fully dissolve lignin until a concentration

173 of 10 wt. \%. Its dissolution efficiency strongly decreases for higher amounts of lignin, as can

174 be observed in Figure 3. The $\mathrm{pH}$ value of each lignin solution was analyzed, and it is reported

175 in Figure 2. The data suggests that the efficiency of the dissolution does not have a direct

176 relationship to the $\mathrm{pH}$ value. In all cases, the samples were prepared in a $\mathrm{pH}$ region below

177 the pKa of the acids, and the dissolution performances are strikingly different. Note that,

178 levulinic, acetic, and propionic acids have a rather similar pKa, ca. 4.8, [23,24]. 

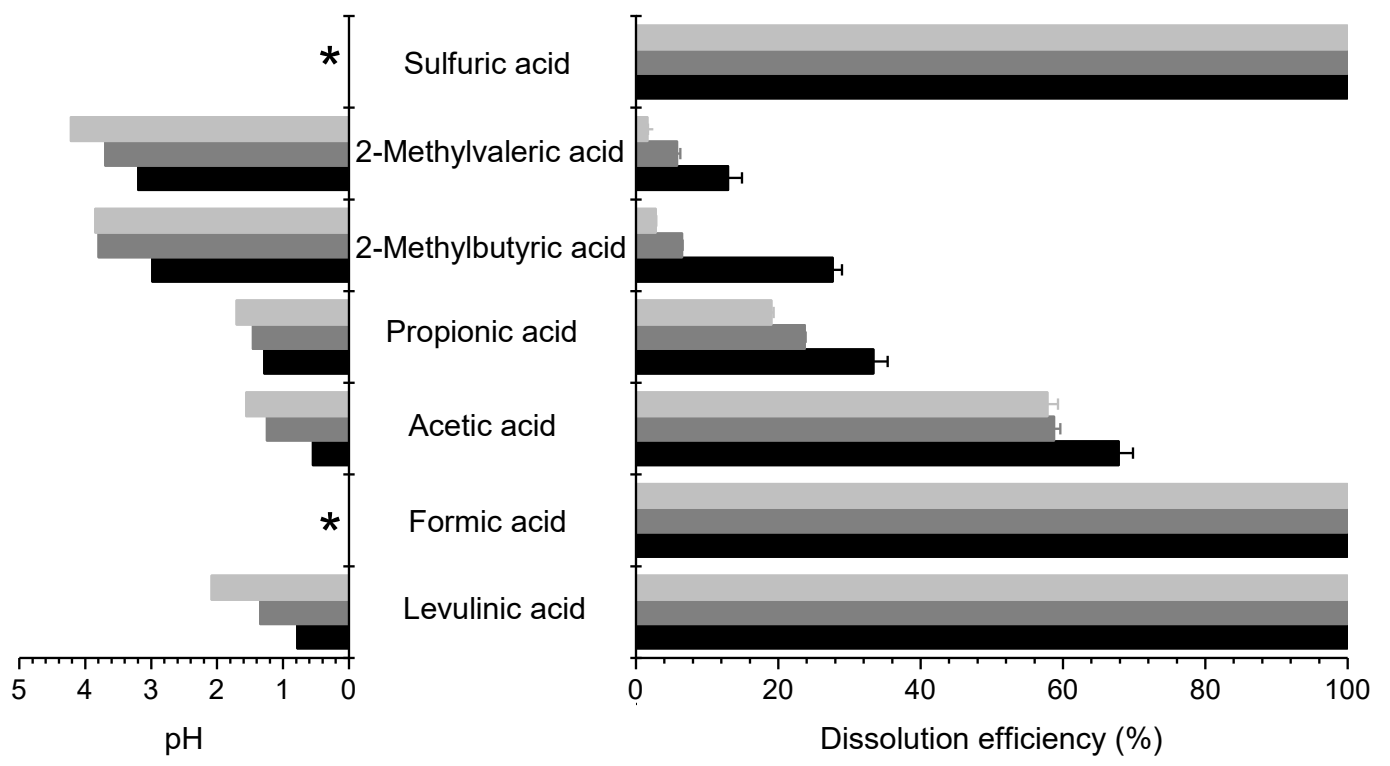

180 Figure 2. $\mathrm{pH}$ and dissolution efficiency of 0.1 (black), 1 (gray) and 10 (light gray) wt. \% kraft lignin dispersed in different acids. Samples marker with "*" show pH below zero.

1

Figure 3. Polarized light micrographs of (a) 10 and (b) 20 wt. \% kraft lignin dissolved in sulfuric 189 acid, at room temperature. The scale bars represent $1 \mathrm{~mm}$.

191 The Kamlet-Taft solvatochromic parameter $\pi^{*}$ was estimated for the different acidic solvents

192 (Figure 4). Data suggest that increasing polarizability of the acidic solvents favors lignin 193 dissolution; acids with high polarizability $\left(\pi^{*}>0.9\right)$ were the most effective to dissolve lignin. 
194 This trend is opposite to what was verified for the alkaline systems [25]. Furthermore, the 195 trend observed for carboxylic acids, in which solubility is favored by a higher $\pi^{*}$ and a smaller 196 carbon chain, is similar to alcohols, where it has already been described that $\pi^{*}$ of methanol 197 is higher than that of 2-propanol [26] and the lignin solubility is higher in methanol $[27,28]$.

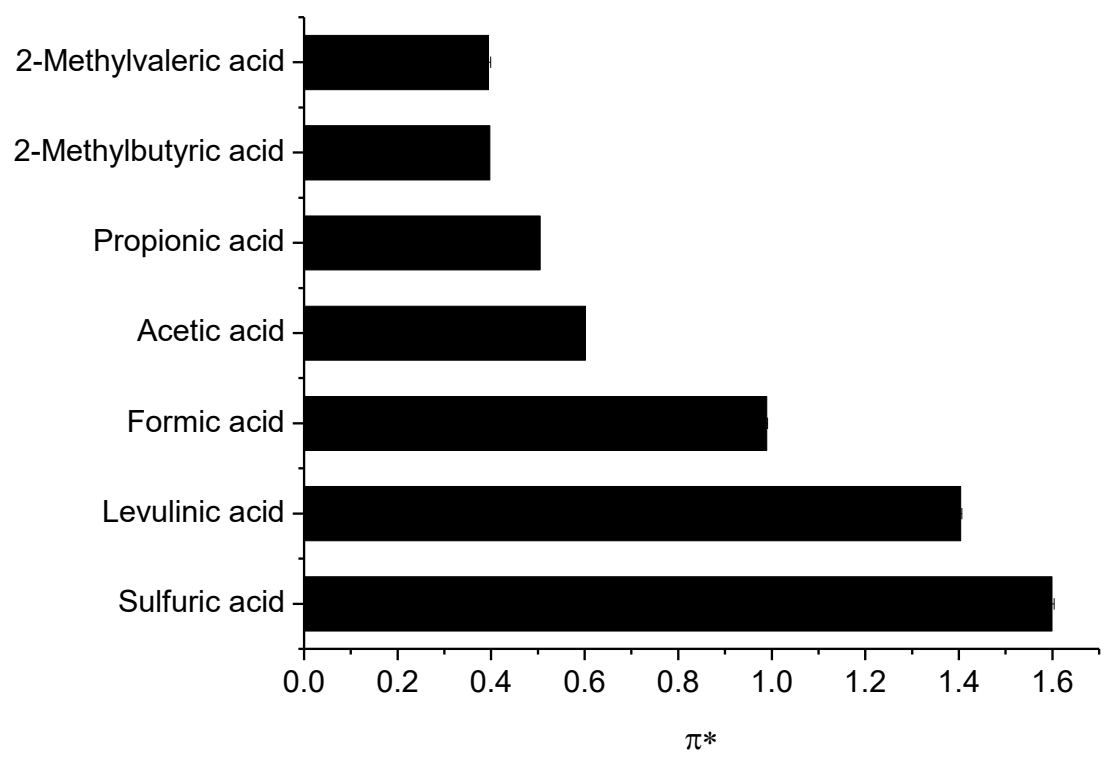

Figure 4. Solvatochromic parameter $\pi^{*}$ for the different acidic solvent systems.

201 A systematic study on the lignin dissolution efficiency with different concentrations of 202 levulinic acid aqueous solutions was performed, using different initial lignin concentrations 203 (i.e., 1, 10, 20 and 30 wt. \%) and temperatures (i.e., 25, 40 and $60^{\circ} \mathrm{C}$ ). As it is possible to 204 observe in Table 1, the temperature rise benefits dissolution in all cases, and this normal 205 thermal effect has been previously reported for other solvent systems $[10,29,30]$.

206 Lignin dissolution was found complete for water concentrations below 40 wt. \% (5.17 M). In 207 this case, it was possible to dissolve up to 40 wt. \% lignin (the same amount using 208 concentrated levulinic acid). For diluted levulinic acid aqueous solutions, above 40 wt. \% 
water, lignin dissolution was incomplete. A similar behavior has been reported for $\gamma$ -

210 valerolactone aqueous system [10]. It was suggested that, for water contents above 50 wt.

$211 \%$, a strong interaction ( $\mathrm{H}$-bonding network) between water and $\gamma$-valerolactone is

212 established decreasing the solvent performance towards lignin. It is reasonable to assume

213 that a similar behavior might be occurring with the levulinic acid system. The water effect

214 and sensitivity in lignin solubility has been observed in other solvent systems, such as ionic

215 liquids (IL); in the IL pyridinium formate, a small amount of water is sufficient to remarkably

216 affect the dissolution [29]. The higher the amount of water the lower the solubility of lignin,

217 being negligible above $50 \%(\mathrm{v} / \mathrm{v})$ of water. In the alkylimidazolium-based IL systems, water

218 tolerance is higher and 30 wt. \% water in the IL still favors lignin solubility [31].

219

220 Table 1. Dissolution efficiency (\%) of kraft lignin in levulinic acid aqueous solutions. Effect of

221 levulinic acid and lignin concentrations at different temperatures.

\begin{tabular}{|c|c|c|c|c|}
\hline \multirow{2}{*}{$\begin{array}{l}\text { Levulinic acid } \\
\qquad(\mathrm{M})\end{array}$} & \multirow{2}{*}{ Kraft lignin (wt. \%) } & \multicolumn{3}{|c|}{ Temperature $(\stackrel{\circ}{ } \mathrm{C})$} \\
\hline & & 25 & 40 & 60 \\
\hline \multirow{4}{*}{0.86} & 1 & $10.93 \pm 0.07$ & $12.0 \pm 0.2$ & $16.5 \pm 0.2$ \\
\hline & 10 & $3.28 \pm 0.04$ & $3.68 \pm 0.02$ & $4.6 \pm 0.5$ \\
\hline & 20 & $2.16 \pm 0.02$ & $2.33 \pm 0.04$ & $2.56 \pm 0.01$ \\
\hline & 30 & $1.75 \pm 0.00$ & $1.79 \pm 0.01$ & $2.06 \pm 0.01$ \\
\hline \multirow{2}{*}{1.72} & 1 & $23.6 \pm 0.9$ & $24.8 \pm 0.2$ & $30.9 \pm 0.8$ \\
\hline & 10 & $7.84 \pm 0.09$ & $8.4 \pm 0.1$ & $8.5 \pm 0.1$ \\
\hline
\end{tabular}




\begin{tabular}{|c|c|c|c|c|}
\hline & 20 & $5.30 \pm 0.05$ & $5.4 \pm 0.2$ & $6.0 \pm 0.1$ \\
\hline & 30 & $3.94 \pm 0.00$ & $3.96 \pm 0.04$ & $4.14 \pm 0.03$ \\
\hline \multirow{4}{*}{2.58} & 1 & $41.70 \pm 0.03$ & $42.9 \pm 0.1$ & $48.5 \pm 0.9$ \\
\hline & 10 & $16.0 \pm 0.3$ & $17.9 \pm 0.3$ & $21.49 \pm 0.04$ \\
\hline & 20 & $10.86 \pm 0.05$ & $11.6 \pm 0.2$ & $11.6 \pm 0.2$ \\
\hline & 30 & $7.4 \pm 0.4$ & $9.9 \pm 0.2$ & $10.58 \pm 0.01$ \\
\hline \multirow{4}{*}{3.44} & 1 & $71.0 \pm 0.7$ & $79 \pm 1$ & $90.11 \pm 0.01$ \\
\hline & 10 & $31 \pm 1$ & $36.5 \pm 0.4$ & $40.2 \pm 0.1$ \\
\hline & 20 & $23.2 \pm 0.4$ & $24.1 \pm 0.1$ & $28.2 \pm 0.8$ \\
\hline & 30 & $15.8 \pm 0.2$ & $17.8 \pm 0.5$ & $18.3 \pm 0.1$ \\
\hline \multirow{4}{*}{4.31} & 1 & $99 \pm 4$ & 100 & 100 \\
\hline & 10 & $61.4 \pm 0.4$ & $67 \pm 3$ & $78.9 \pm 0.9$ \\
\hline & 20 & $63 \pm 2$ & $61.4 \pm 0.3$ & $75.2 \pm 0.5$ \\
\hline & 30 & $44 \pm 2$ & $49 \pm 3$ & $65 \pm 3$ \\
\hline \multirow{4}{*}{5.17} & 1 & 100 & 100 & 100 \\
\hline & 10 & 100 & 100 & 100 \\
\hline & 20 & 100 & 100 & 100 \\
\hline & 30 & 100 & 100 & 100 \\
\hline
\end{tabular}

224 The water content effect on the solvatochromic parameter $\pi *$ was also evaluated (Figure 5).

225 Overall, this parameter was found to decrease with increasing water content. Above 60 wt. 
$226 \%$ of water (4.31 M levulinic acid), the $\pi^{*}$ approaches the $\pi^{*}$ value of pure water. As we have

227 previously observed, this also coincides with the point where the dissolution efficiency is

228 seriously compromised.

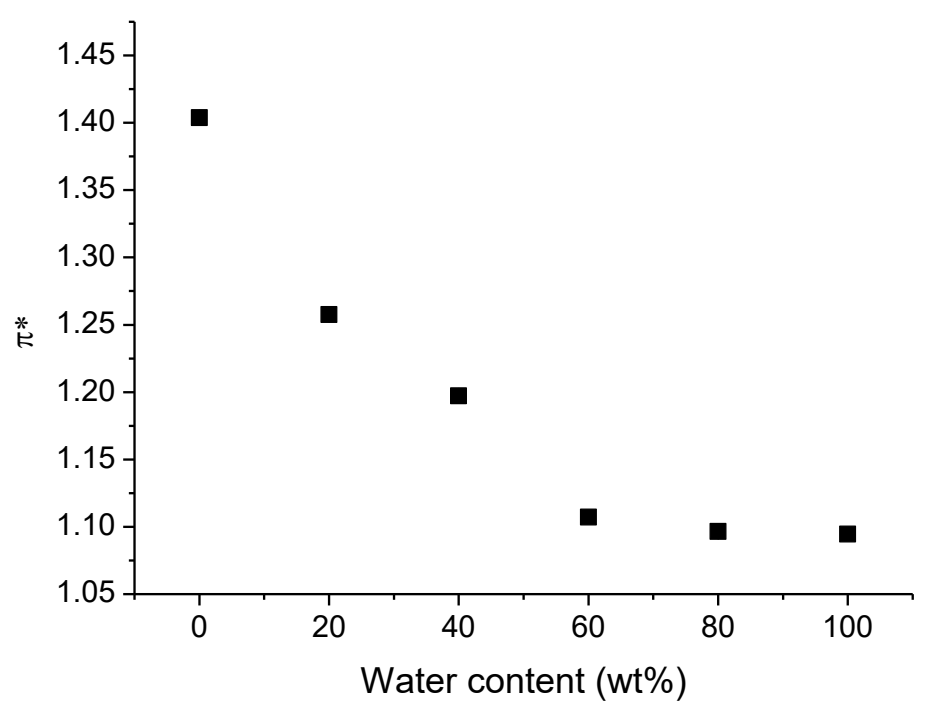

230 Figure 5. Solvatochromic parameter $\pi^{*}$ of levulinic acid aqueous solutions with different 231 water contents.

232

\subsection{Rheology}

234 Using a levulinic acid aqueous binary system has several obvious advantages. Not only the 235 solvent system becomes inexpensive and more environmentally friendly, but the viscosity 236 significantly decreases benefiting its manipulation. Remarkably, by adding water, it is 237 possible to decrease the viscosity by, ca. one order of magnitude without seriously 238 compromising the dissolution efficiently of levulinic acid (Figure 6). The lower solution 239 viscosity has benefits in some applications, such as in the production of lignin-based 240 polyurethane foams, where the high viscosity of commercial lignins is a serious issue [32] or 
241 in development of lignin-based composite materials, where the typical high viscosity limits,

242 for instance, the potential of grafting [33].

243

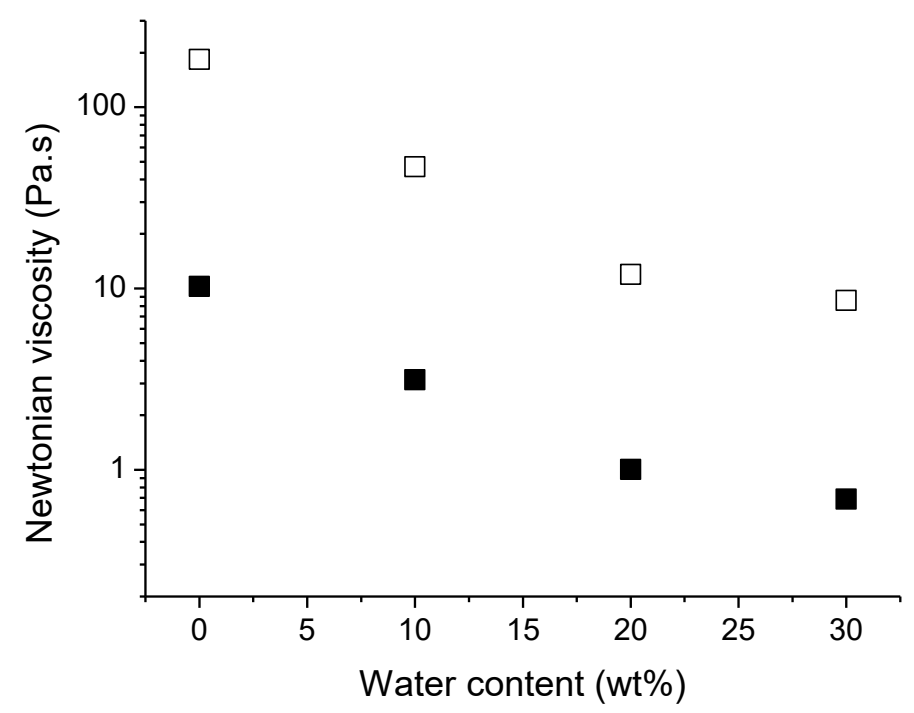

244 Figure 6. Newtonian viscosity of solutions with 30 (full symbols) and 40 (empty symbols) wt.

$245 \%$ kraft lignin dissolved in levulinic acid with different water concentrations. Note that in all 246 cases lignin dissolution is complete.

247

\subsection{Lignin characterization after solubilization in levulinic and formic acids}

249 After full dissolution in levulinic acid, lignin was precipitated and recovered by adding excess

250 water. The regenerated material was lyophilized and characterized using FTIR, TGA and SEM.

251 For comparison, the same procedure was performed with formic acid which, in the same

252 conditions, has a similar dissolution performance to levulinic acid (see Figure 2 for details). 
255 The FTIR spectra of the initial and regenerated lignins, after dissolution in levulinic and formic

256 acids, are shown in Figure 7 and 8 . The ratio of absorbances $A_{x} / A_{1600,}$ where $A x$ represents

257 each band identified and numbered in Figures 7 and 8, allows a comparative assessment of

258 the effect of type of acids and concentration on the lignin structure $[38,39]$. This analysis was

259 performed for all observed vibrational modes, and the data is summarized in tables S1 and

260 S2. The reference band (R) can be attributed to aromatic skeletal vibrations plus the $\mathrm{C}=\mathrm{O}$

261 stretch [34]. The most significant difference in spectra is the appearance of a well-defined

262 band at $1705-1712 \mathrm{~cm}^{-1}(\# 1)$. This band is assigned to the $\mathrm{C}=\mathrm{O}$ stretching in unconjugated

263 ketone, carbonyl and in ester groups [34]. In electrochemical applications, the increase

264 content of carbonyl groups is an advantage for enhanced protonic and electrical

265 conductivities in active materials [35]. Its relative intensity is higher using formic acid than

266 when using levulinic acid in the dissolution process. For the diluted levulinic acid, the lower

267 the acid concentration, the less intense the band is (Figure 9). The band \#2, at $1510-1512$

$268 \mathrm{~cm}^{-1}$, is assigned to the aromatic skeleton vibration [34]. The absence of a significant

269 decrease in the relative intensity of this band suggests that the dissolution and regeneration

270 processes do not affect significantly the integrity of the aromatic rings [36]. The decrease of

271 the relative absorbance in the bands \#3 (i.e., $\mathrm{C}-\mathrm{H}$ bending of the methyl or methylene groups

272 [37]) and \#4 (i.e., aromatic skeletal vibration combined with the $\mathrm{C}-\mathrm{H}$ asymmetric deformation

273 of the methyl groups [34]) for all regenerated lignins, suggests the possible hydrolysis of the

274 methoxy groups during the dissolution or regeneration process [36]. In addition, the

275 intensity decreases of the C-O deformation in primary (\#11) and secondary (\#10) alcohols,

276 suggests a degradation of the aliphatic chain of the phenyl propane units for all regenerated 
277 lignins. This reaction also contributes for the enhancement of the relative intensity of the $278 \mathrm{C}=\mathrm{O}$ band [36]. Differences between levulinic and formic acid are observed regarding the 279 cleavage of ether bonds. Using levulinic acid, an increase of the vibrational band assigned to 280 the phenolic hydroxyl groups occurs[29] (\#5) while a decrease of the intensity of the ether281 O bands [29] (\#9), is observed. Such decrease is most likely related to the cleavage of these 282 linkages, mainly $\beta-0-4$, the most abundant linkage in lignin and one of the most easily cleaved 283 bonds [40]. Using formic acid, an opposite effect was observed.

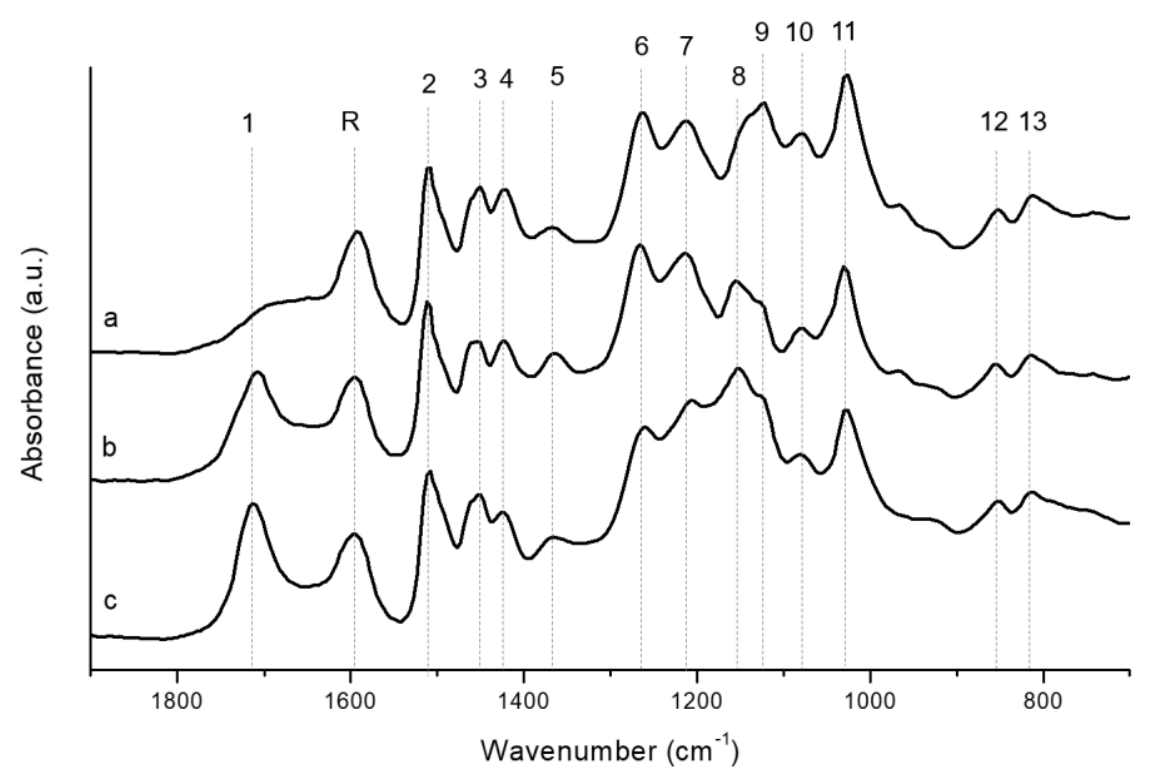

286 Figure 7. Normalized FTIR spectra of: (a) initial lignin and recovered lignin after dissolution in 287 (b) levulinic acid and (c) formic acid. The vibration mode assignment and semiquantitative 288 analysis is performed in Table S1 and discussed in the text. 


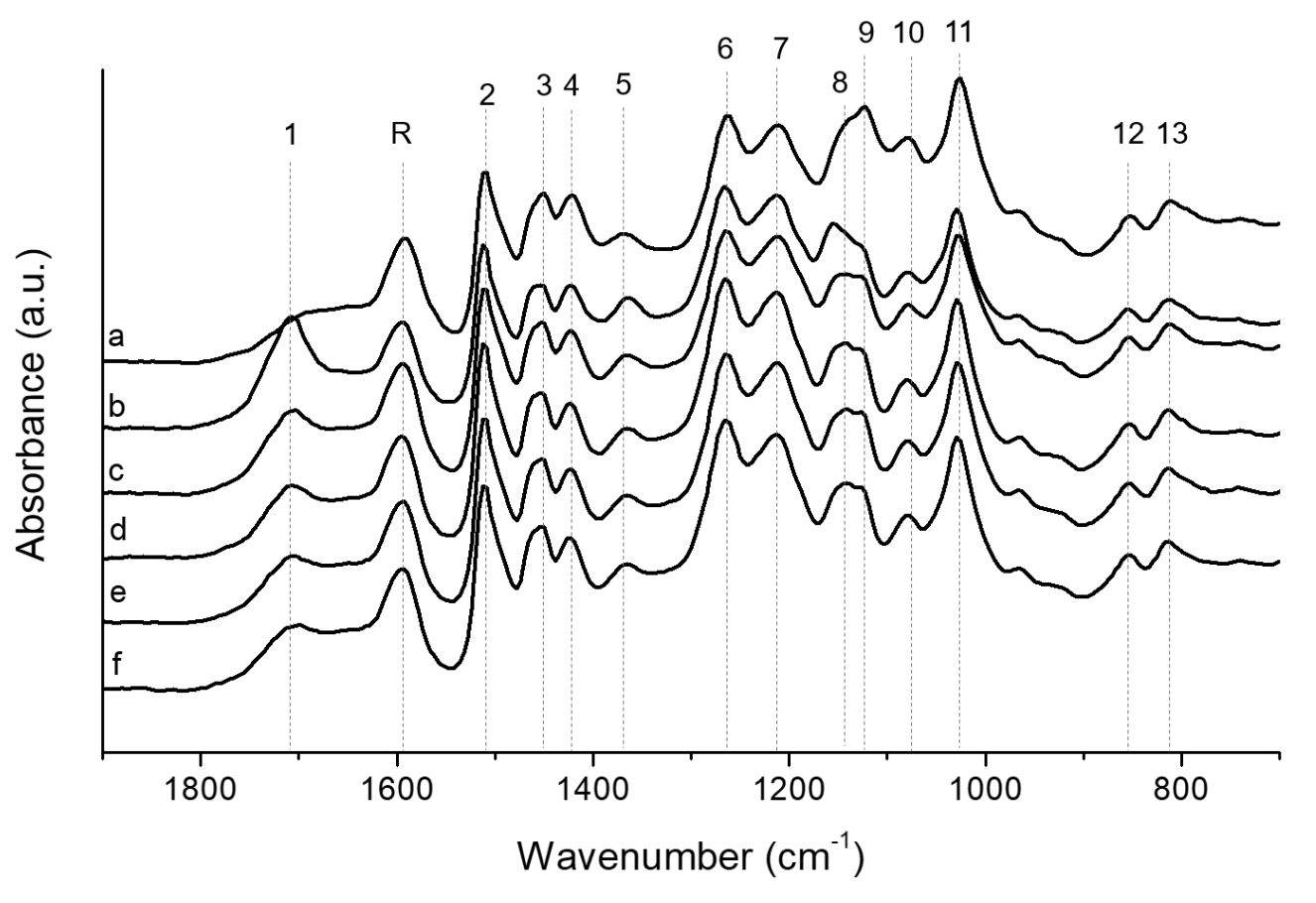

291 Figure 8. Normalized FTIR spectra of: (a) initial lignin; recovered lignin after dissolution in (b)

292 concentrated levulinic acid and levulinic acid aqueous solutions of (c) 7.75 M, (d) 6.89 M, (e)

$2936.03 \mathrm{M}$, and ( $\mathrm{f}$ ) $5.17 \mathrm{M}$. The vibration mode assignment and semiquantitative analysis is 294 performed in Table S2 and discussed in the text. 


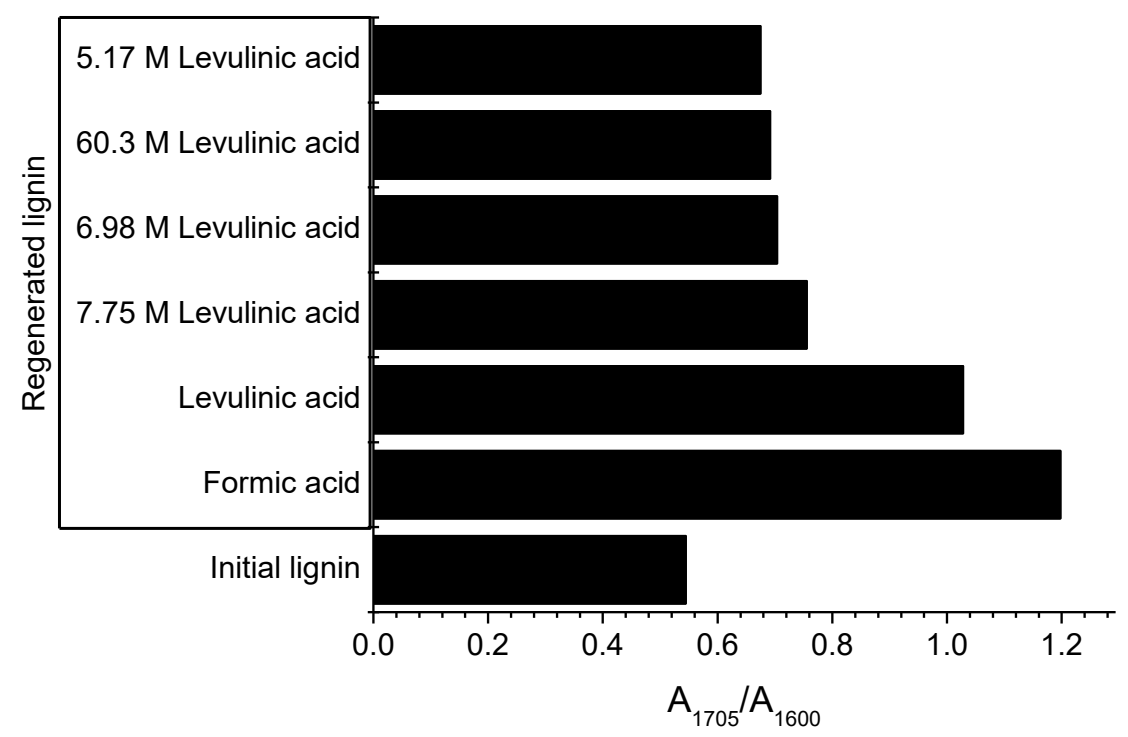

Figure 92 . Semi-quantitative analysis $\left(A_{1705} / A_{1600}\right)$ of initial and regenerated lignins.

\subsubsection{Thermogravimetric analysis}

299 The thermal decomposition of native and regenerated lignins was performed and compared

300 in Figure 10. From the analysis of thermograms and corresponding DTGs, it can be observed

301 that some weight loss occurs at temperatures between $80-150 \stackrel{\circ}{ } \mathrm{C}$, corresponding to the 302 evaporation of physically adsorbed water [41]. In the raw lignin and regenerated material 303 after the dissolution in levulinic acid, this step occurs until $100^{\circ} \mathrm{C}$. In the case of the lignin 304 regenerated after being dissolved in formic acid, this step is verified until $150{ }^{\circ} \mathrm{C}$, most likely 305 due to the volatilization of low molecular weight compounds in addition to the removal of 306 water [29]. The main degradation step occurs at a maximum temperature, $T_{m}$, corresponding 307 to the maximum rate of degradation, $383{ }^{\circ} \mathrm{C}$ for the initial lignin. However, for the 308 regenerated lignin previously dissolved in formic and levulinic acids, the $T_{\mathrm{m}}$ decreases to 377 and $367^{\circ} \mathrm{C}$, respectively. These values are in agreement with those reported in literature, 
where most lignins present their maximum weight loss rate between 300 and $400{ }^{\circ} \mathrm{C}$ [41].

311 Neverteless, , these results also suggest that lignin becomes less structured upon

312 regeneration. This effect is more pronounced when using levulinic acid, due to the cleavage

313 of the ether linkages discussed in the FTIR analysis. After heating to $900^{\circ} \mathrm{C}$, the regenerated

314 material obtained after dissolution in formic and levulinic acids, have a portion unvolatilized

315 equivalent to ca. 28 and 33 wt. \%, respectively. Since in the initial kraft lignin, ca. 29 wt. \%

316 remains unvolatilized, this suggests that some condensed aromatic structures may be

317 formed during the dissolution and regeneration processes[43].
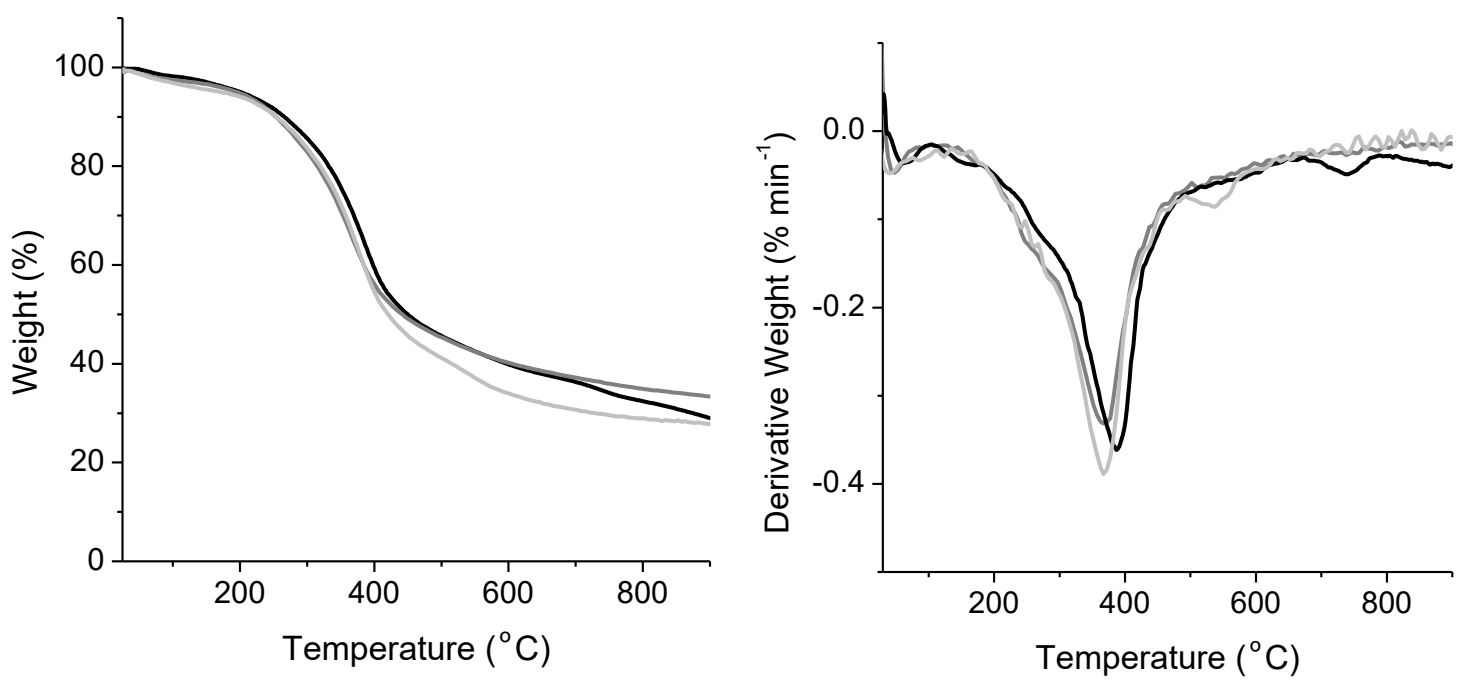

320 Figure 10. TGA (left) and the correspondent DTG (right) of the regenerated material obtained

321 from the dissolution of 40 wt. \% kraft lignin in formic (light gray line) and levulinic acid (gray

322 line). The initial raw kraft lignin is represented with a black line.

323

\subsubsection{Scanning electron microscopy}


325 The dissolution and regeneration effects on the morphology and microstructure of the

326 regenerated materials were analyzed by SEM and compared to the initial raw kraft lignin

327 (Figure 11). The initial lignin sample presents particles with different shapes and sizes, which

328 are typical for this type of lignin [44]. Their average size is in accordance with previous works,

329 ca. 1-2 $\mu \mathrm{m}$ [45]. On the other hand, the regenerated lignins present some differences; lignin

330 dissolved in formic acid (Figures 11c and 11d) have a more heterogeneous structure, with

331 larger and very smaller particles, in comparison to the initial lignin. On the other hand, when

332 dissolved in levulinic acid (Figures 11e and 11f) the structure is more homogeneous, but the

333 particles are smaller when compared to raw lignin. Most likely, this morphological effect is

334 due to higher degree of depolymerization, as suggested by the FTIR and TG analysis.

335

336
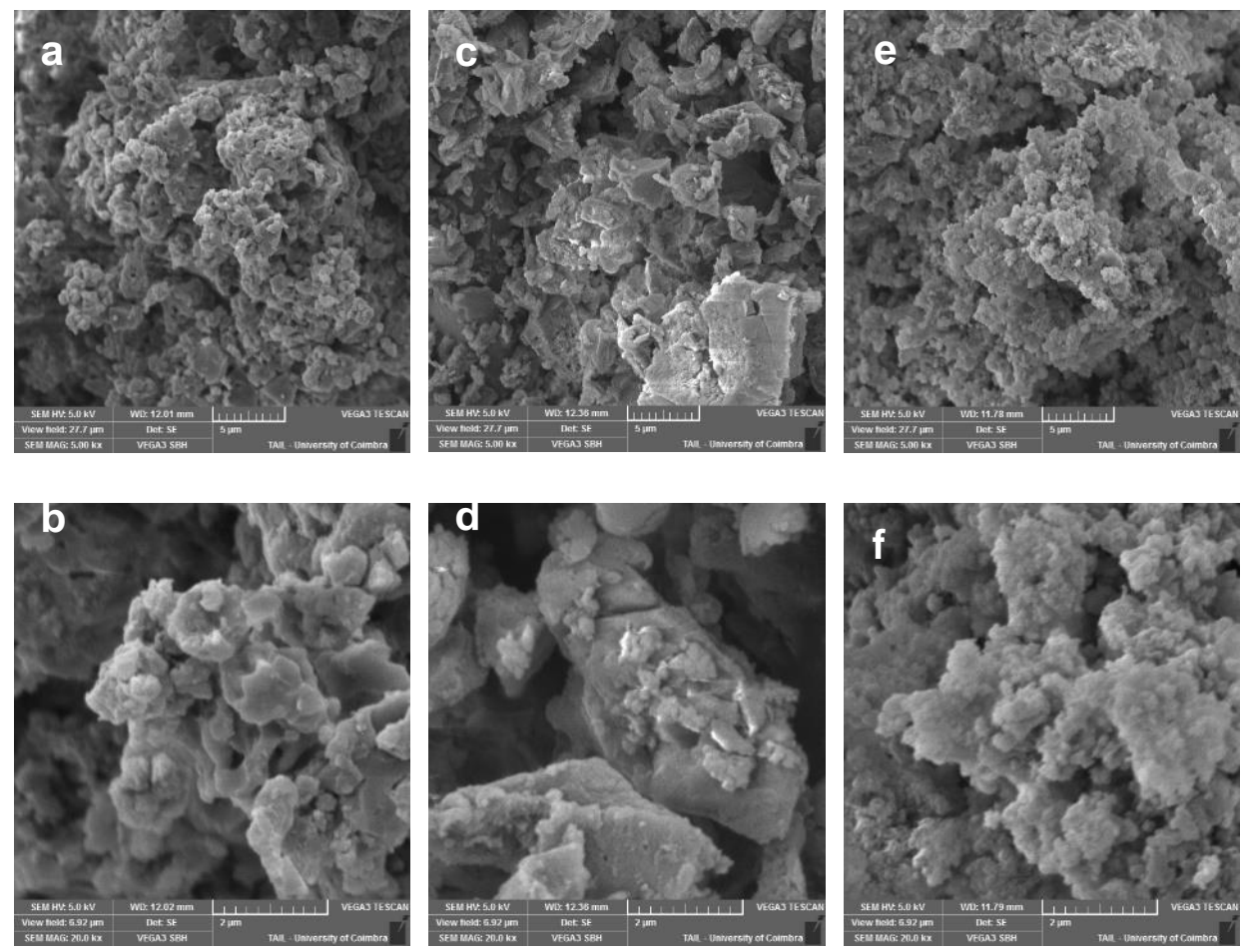
338 Figure 11. SEM images at different magnifications of a, b) initial raw lignin; $c$, d) lignin

339 regenerated after dissolution in formic acid; and e, f) lignin regenerated after dissolution in

340 levulinic acid. Magnification x 5,000 and 20,000.

\section{Conclusions}

343 This work reports, for the first time, the use of levulinic acid as a sustainable solvent of 344 superior performance for lignin dissolution. In addition to the benefits of using an 345 environmentally friendly solvent when compared to the harmful systems commonly used,

346 levulinic acid is still quite efficient upon water dilution up to a concentration of ca. $5.17 \mathrm{M}$.

347 Lignin dissolution seems to correlate well with solvent polarizability. The addition of water

348 greatly reduces the viscosity of the solution, which might facilitate large-scale processing, 349 such as mixing, pumping or extrusion from containers, and also makes the solvent 350 inexpensive. The lignins dissolved in levulinic can be regenerated by the addition of excess

351 water. These regenerated materials have different contents of functional groups, being the 352 most important difference found in the $\mathrm{C}=\mathrm{O}$ content. Besides that, the FTIR analysis suggests 353 the occurrence of partial methoxy groups hydrolysis, degradation of aliphatic chain of 354 phenylpropane and cleavage of ether bonds. Nevertheless, these changes do not result in 355 significant differences in terms of thermal stability and morphological characteristics, when

356 compared to the initial raw material or lignin dissolved in formic acid. Overall, this work 357 characterizes a novel and ecofriendly aqueous-based solvent for lignin, which might be very 358 interesting for its processing at the industrial level, creating opportunities for the 359 development of new applications for lignin that have not been exploited so far due to its 
360 difficult dissolution. Additionally, this work also sheds light on the mechanisms involved in

361 lignin dissolution highlighting the role of solvent polarizability.

362

\section{Acknowledgements}

364 This work was supported by funding from the Portuguese Foundation for Science and

365 Technology (FCT) through the projects PTDC/AGR-TEC/4814/2014, PTDC/ASP-

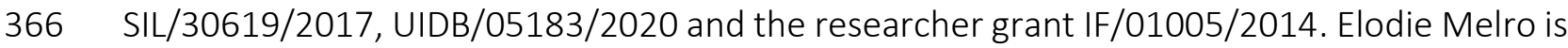
367 grateful for the PhD grant (SFRH/BD/132835/2017) from FCT. The CQC is supported by FCT

368 through the project PEstOE/QUI/UI0313/2013.

369

370 References

371 [1] Y. Pu, N. Jiang, A.J. Ragauskas, lonic liquid as a green solvent for lignin, J. Wood Chem.

$372 \quad$ Technol. (2007). doi:10.1080/02773810701282330.

373 [2] D. Kai, M.J. Tan, P.L. Chee, Y.K. Chua, Y.L. Yap, X.J. Loh, Towards lignin-based functional 374 materials in a sustainable world, Green Chem. 18 (2016) 1175-1200. 375 doi:10.1039/c5gc02616d.

376 [3] M. Norgren, H. Edlund, Lignin: Recent advances and emerging applications, Curr. Opin. Colloid Interface Sci. (2014). doi:10.1016/j.cocis.2014.08.004.

378 [4] M. Norgren, B. Lindström, Dissociation of phenolic groups in kraft lignin at elevated 379 temperatures, Holzforschung. (2000). doi:10.1515/HF.2000.088.

380 [5] M. Norgren, H. Edlund, L. Wågberg, B. Lindström, G. Annergren, Aggregation of kraft 381 lignin derivatives under conditions relevant to the process, part I: Phase behaviour, 
Colloids Surfaces A Physicochem. Eng. Asp. (2001). doi:10.1016/S09277757(01)00753-1.

384 [6] M. Norgren, H. Edlund, L. Wågberg, Aggregation of lignin derivatives under alkaline 385 conditions. Kinetics and aggregate structure, Langmuir. (2002).

387 [7] M. Norgren, B. Lindström, Physico-chemical characterization of a fractionated kraft 388 doi:10.1021/la011627d.

[9] M. Norgren, H. Edlund, lon specific differences in salt induced precipitation of kraft 393 lignin, Nord. Pulp Pap. Res. J. (2003). doi:10.3183/npprj-2003-18-04-p400-403.

[10] Z. Xue, X. Zhao, R.C. Sun, T. Mu, Biomass-derived v-valerolactone-based solvent

[8] M. Norgren, H. Edlund, Stabilisation of kraft lignin solutions by surfactant additions, Colloids Surfaces A Physicochem. Eng. Asp. (2001). doi:10.1016/S09277757(01)00806-8. systems for highly efficient dissolution of various lignins: Dissolution behavior and mechanism study, ACS Sustain. Chem. Eng. (2016). doi:10.1021/acssuschemeng.6b00639.

[11] X.R. Shen, D.Z. Xia, Y.X. Xiang, J.G. Gao, Y-valerolactone (GVL) as a bio-based green solvent and ligand for iron-mediated AGET ATRP, E-Polymers. (2019). doi:10.1515/epoly-2019-0033.

[12] D.M. Alonso, S.G. Wettstein, J.A. Dumesic, Gamma-valerolactone, a sustainable platform molecule derived from lignocellulosic biomass, Green Chem. (2013). doi:10.1039/c3gc37065h. 
[13] E. Melro, L. Alves, F.E. Antunes, B. Medronho, A brief overview on lignin dissolution, J. Mol. Liq. 265 (2018). doi:10.1016/j.molliq.2018.06.021.

406 [14] H. Singh, N. Iyengar, R. Yadav, A. Rai, A.K. Sinha, Facile conversion of levulinic acid to -valerolactone using a high surface area magnetically separable $\mathrm{Ni} / \mathrm{NiO}$ catalyst,

[15] L. Lomba, B. Giner, I. Bandrés, C. Lafuente, M.R. Pino, Physicochemical properties of Sustain. Energy Fuels. (2018). doi:10.1039/c8se00274f. green solvents derived from biomass, Green Chem. (2011). doi:10.1039/c0gc00853b. Biofuels, Bioprod. Biorefining. (2011). doi:10.1002/bbb.267.

C. CHANG, X. MA, P. CEN, Kinetics of Levulinic Acid Formation from Glucose Decomposition at High Temperature, Chinese J. Chem. Eng. (2006). doi:10.1016/s1004-9541(06)60139-0. doi:10.1021/acs.jced.6b00164.

C. Chang, P. Cen, X. Ma, Levulinic acid production from wheat straw, Bioresour. Technol. (2007). doi:10.1016/j.biortech.2006.03.031.

422 [20] M.J. Kamlet, J.L. Abboud, R.W. Taft, The Solvatochromic Comparison Method. 6. The $\pi^{*}$ Scale of Solvent Polarities1, J. Am. Chem. Soc. (1977). doi:10.1021/ja00460a031.

424 [21] D. González-Arjona, G. López-Pérez, M.M. Domínguez, A.G. González, 425 Solvatochromism: A Comprehensive Project for the Final Year Undergraduate 
427 [22] A. Duval, F. Vilaplana, C. Crestini, M. Lawoko, Solvent screening for the fractionation 428 of industrial kraft lignin, Holzforschung. 70 (2016) 11-20. doi:10.1515/hf-2014-0346.

429 [23] T.H. Eberlein, Essentials of Organic Chemistry: For Students of Pharmacy, Medicinal 430 Chemistry, and Biological Chemistry (Paul M. Dewick), J. Chem. Educ. (2008). 431 doi:10.1021/ed085p204.

432 [24] A.A. Sibirny, Biotechnology of yeasts and filamentous fungi, 2017. doi:10.1007/978-3$433 \quad 319-58829-2$.

434 [25] E. Melro, A. Filipe, D. Sousa, A.J.M. Valente, A. Romano, F.E. Antunes, B. Medronho, 435 Dissolution of kraft lignin in alkaline solutions, Int. J. Biol. Macromol. (2020). doi:10.1016/j.ijbiomac.2020.01.153.

437 [26] W.J. Cheong, P.W. Carr, Kamlet-Taft $\pi^{*}$ Polarizability/Dipolarity of Mixtures of Water 438 with Various Organic Solvents, Anal. Chem. (1988). doi:10.1021/ac00159a018.

439 [27] J. Sameni, S. Krigstin, M. Sain, Solubility of Lignin and Acetylated Lignin in Organic $440 \quad$ Solvents, BioResources. (2017). doi:10.15376/biores.12.1.1548-1565.

441 [28] J. Sun, T. Dutta, R. Parthasarathi, K.H. Kim, N. Tolic, R.K. Chu, N.G. Isern, J.R. Cort, B.A. 442 Simmons, S. Singh, Rapid room temperature solubilization and depolymerization of 443 polymeric lignin at high loadings, Green Chem. (2016). doi:10.1039/c6gc02258h.

444 [29] T. Rashid, C.F. Kait, I. Regupathi, T. Murugesan, Dissolution of kraft lignin using Protic 445 Ionic Liquids and characterization, Ind. Crops Prod. (2016). 446 doi:10.1016/j.indcrop.2016.02.017.

447 [30] D. Glas, C. Van Doorslaer, D. Depuydt, F. Liebner, T. Rosenau, K. Binnemans, D.E. De 
448

449

450

451

452

453

454

455

456

457

458

459

460

461

462

463

464

465

466

467

468

469

Vos, Lignin solubility in non-imidazolium ionic liquids, J. Chem. Technol. Biotechnol. (2015). doi:10.1002/jctb.4492.

[31] Y. Wang, L. Wei, K. Li, Y. Ma, N. Ma, S. Ding, L. Wang, D. Zhao, B. Yan, W. Wan, Q. Zhang, X. Wang, J. Wang, H. Li, Lignin dissolution in dialkylimidazolium-based ionic liquid-water mixtures, Bioresour. Technol. (2014). doi:10.1016/j.biortech.2014.08.020.

[32] J. Bernardini, P. Cinelli, I. Anguillesi, M.B. Coltelli, A. Lazzeri, Flexible polyurethane foams green production employing lignin or oxypropylated lignin, Eur. Polym. J. 64 (2015) 147-156. doi:10.1016/j.eurpolymj.2014.11.039.

[33] W. Thielemans, E. Can, S.S. Morye, R.P. Wool, Novel applications of lignin in composite materials, J. Appl. Polym. Sci. (2002). doi:10.1002/app.2247.

[34] O. Faix, Classification of Lignins from Different Botanical Origins by FT-IR Spectroscopy, Holzforschung. (1991). doi:10.1515/hfsg.1991.45.s1.21.

[35] Ł. Klapiszewski, T.J. Szalaty, B. Kurc, M. Stanisz, B. Zawadzki, A. Skrzypczak, T. Jesionowski, Development of Acidic Imidazolium Ionic Liquids for Activation of Kraft Lignin by Controlled Oxidation: Comprehensive Evaluation and Practical Utility, Chempluschem. (2018). doi:10.1002/cplu.201800123.

[36] A.A.M.A. Nada, M.A. Yousef, K.A. Shaffei, A.M. Salah, Infrared spectroscopy of some treated lignins, Polym. Degrad. Stab. (1998). doi:10.1016/S0141-3910(97)00273-5.

[37] S. Sathawong, W. Sridach, K.A. Techato, Lignin: Isolation and preparing the lignin based hydrogel, J. Environ. Chem. Eng. (2018). doi:10.1016/j.jece.2018.05.008.

[38] A. Tawansi, A. El-Khodary, M.M. Abdelnaby, A study of the physical properties of FeCl3 
filled PVA, Curr. Appl. Phys. (2005). doi:10.1016/j.cap.2004.06.026.

471 [39] A. Papancea, A.J.M. Valente, S. Patachia, Diffusion and sorption studies of dyes through PVA cryogel membranes, J. Appl. Polym. Sci. (2010). doi:10.1002/app.30983.

473 [40] P.K. Mishra, A. Ekielski, The self-assembly of lignin and its application in nanoparticle synthesis: A short review, Nanomaterials. (2019). doi:10.3390/nano9020243.

475 [41] H. Yoshida, R. Mörck, K.P. Kringstad, H. Hatakeyama, Fractionation of kraft lignin by 476 fractions, Holzforschung. 41 (1987) 171-176. doi:10.1515/hfsg.1987.41.3.171.

[42] R.C. Sun, J. Tomkinson, G. Lloyd Jones, Fractional characterization of ash-AQ lignin by successive extraction with organic solvents from oil palm EFB fibre, Polym. Degrad. Stab. (2000). doi:10.1016/S0141-3910(99)00174-3.

[43] A. Tejado, C. Peña, J. Labidi, J.M. Echeverria, I. Mondragon, Physico-chemical characterization of lignins from different sources for use in phenol-formaldehyde resin synthesis, Bioresour. Technol. (2007). doi:10.1016/j.biortech.2006.05.042.

[44] M. Wysokowski, Ł. Klapiszewski, D. Moszyński, P. Bartczak, T. Szatkowski, I. Majchrzak, K. Siwińska-Stefańska, V. V. Bazhenov, T. Jesionowski, Modification of chitin with kraft ions, Mar. Drugs. (2014). doi:10.3390/md12042245.

Q. Yan, J. Li, J. Zhang, Z. Cai, Thermal decomposition of Kraft Lignin under Gas atmospheres of argon, hydrogen, and carbon dioxide, Polymers (Basel). (2018). doi:10.3390/polym10070729. 
493

\section{$494 \quad$ Table S1}

495 FTIR semiquantitative analysis of initial lignin and regenerated lignins obtained after 496 dissolution in levulinic and formic acid.

\begin{tabular}{|c|c|c|c|c|c|c|}
\hline \multirow{3}{*}{ № } & \multirow{2}{*}{\multicolumn{2}{|c|}{ Kraft Lignin }} & \multicolumn{4}{|c|}{ Regenerated lignin } \\
\hline & & & \multicolumn{2}{|c|}{ Levulinic acid } & \multicolumn{2}{|c|}{ Formic acid } \\
\hline & Band & Ax/A1600 & Band & Ax/A1600 & Band & Ax/A1600 \\
\hline 1 & \multicolumn{2}{|c|}{ Shoulder } & 1709 & 1.0284 & 1712 & 1.1980 \\
\hline $\mathrm{R}$ & 1593 & 1.0000 & 1593 & 1.0000 & 1595 & 1.000 \\
\hline 2 & 1510 & 1.4000 & 1512 & 1.4102 & 1510 & 1.4117 \\
\hline 3 & 1450 & 1.2761 & 1453 & 1.1936 & 1450 & 1.2597 \\
\hline 4 & 1423 & 1.2598 & 1423 & 1.1964 & 1423 & 1.1419 \\
\hline 5 & 1369 & 1.0246 & 1365 & 1.1297 & 1365 & 0.9763 \\
\hline 6 & 1261 & 1.7415 & 1265 & 1.7145 & 1257 & 1.6971 \\
\hline 7 & 1211 & 1.6863 & 1211 & 1.6682 & 1207 & 1.8733 \\
\hline 8 & 1142 & 1.7063 & 1153 & 1.5187 & 1153 & 2.0852 \\
\hline 9 & 1122 & 1.8015 & 1126 & 1.4058 & 1126 & 1.9083 \\
\hline 10 & 1080 & 1.6115 & 1080 & 1.2648 & 1080 & 1.5233 \\
\hline 11 & 1026 & 1.9720 & 1029 & 1.5989 & 1026 & 1.8130 \\
\hline 12 & 852 & 1.1367 & 856 & 1.0691 & 852 & 1.2165 \\
\hline 13 & 814 & 1.2230 & 814 & 1.1197 & 814 & 1.2725 \\
\hline
\end{tabular}

497 
499 FTIR semiquantitative analysis of regenerated lignins previously dissolved in levulinic acid 500 aqueous solutions of different concentrations.

\begin{tabular}{|c|c|c|c|c|c|c|c|c|}
\hline \multirow{3}{*}{ № } & \multicolumn{8}{|c|}{ Concentration of aqueous levulinic acid solutions } \\
\hline & \multicolumn{2}{|c|}{$7.75 \mathrm{M}$} & \multicolumn{2}{|c|}{$6.98 \mathrm{M}$} & \multicolumn{2}{|c|}{$6.03 \mathrm{M}$} & \multicolumn{2}{|c|}{$5.17 \mathrm{M}$} \\
\hline & Band & $\mathrm{Ax} / \mathrm{A} 1600$ & Band & Ax/A1600 & Band & Ax/A1600 & Band & $\mathrm{Ax} / \mathrm{A} 1600$ \\
\hline 1 & 1705 & 0.7560 & 1705 & 0.7039 & 1705 & 0.6920 & 1705 & 0.6752 \\
\hline $\mathrm{R}$ & 1593 & 1.0000 & 1593 & 1.0000 & 1593 & 1.0000 & 1593 & 1.0000 \\
\hline 2 & 1512 & 1.3896 & 1512 & 1.5545 & 1512 & 1.4660 & 1512 & 1.4776 \\
\hline 3 & 1454 & 1.2140 & 1454 & 1.2633 & 1454 & 1.2369 & 1454 & 1.2427 \\
\hline 4 & 1423 & 1.1710 & 1423 & 1.2022 & 1423 & 1.1834 & 1423 & 1.1771 \\
\hline 5 & 1365 & 1.0415 & 1365 & 1.0477 & 1365 & 1.0338 & 1365 & 1.0251 \\
\hline 6 & 1265 & 1.69808 & 1265 & 1.9444 & 1265 & 1.8308 & 1265 & 1.8519 \\
\hline 7 & 1212 & 1.6611 & 1212 & 1.8605 & 1212 & 1.7822 & 1212 & 1.7659 \\
\hline 8 & 1142 & 1.4671 & 1142 & 1.5618 & 1142 & 1.5233 & 1142 & 1.4932 \\
\hline 9 & 1126 & 1.4557 & 1126 & 1.5240 & 1126 & 1.5059 & 1126 & 1.4682 \\
\hline 10 & 1080 & 1.3050 & 1080 & 1.3409 & 1080 & 1.3432 & 1080 & 1.3061 \\
\hline 11 & 1030 & 1.6658 & 1030 & 1.8235 & 1030 & 1.7837 & 1030 & 1.7562 \\
\hline 12 & 852 & 1.1362 & 852 & 1.0803 & 852 & 1.1014 & 852 & 1.0806 \\
\hline 13 & 814 & 1.2062 & 814 & 1.1594 & 814 & 1.1882 & 814 & 1.1594 \\
\hline
\end{tabular}

501

502 